\title{
Toxidade do pólen de Ferdinandusa paraensis Ducke (Rubiaceae) para abelhas Apis mellifera L. (Hymenoptera, Apidae) em Roraima, Brasil, região Amazônica
}

\author{
Sílvio José Reis da Silva ${ }^{1}$, Maria Lúcia $\mathrm{Absy}^{2}$, Adriana $\mathrm{Flach}^{3}$, Raquel Oliveira das \\ Neves $^{4}$ \& Osmar Malaspina ${ }^{5}$
}

1. Instituto de Amparo a Ciência, Tecnologia e Inovação do Estado de Roraima, Museu Integrado de Roraima, Av. Brigadeiro Eduardo Gomes, 2868 Parque Anauá CEP: 69.305-010, Boa Vista, Roraima.

E-mail: silviojosereisdasilva@yahoo.com.br

2. Instituto Nacional de Pesquisas da Amazônia, Coordenação em Botânica, Av. André Araújo 2936, Bairro: Petrópolis, CEP: 69.060-001, Manaus, Amazonas. E-mail: luciabsy@inpa.gov.br.

3. Universidade Federal de Roraima, Departamento de Química, Boa Vista, Roraima. E-mail: aflach@gmail.com

4. Faculdades Cathedral, acadêmica do curso de Ciências Biológicas, Rua Canuto Chaves, 293. Boa Vista, Roraima.

5. Universidade Estadual Paulista, Centro de Estudos de Insetos Sociais UNESP/Rio Claro, São Paulo.

E-mail: malaspin@life.rc.unesp.br

Recebido em: 09/06/2015 Aceito em: 28/08/2015 Publicado online em PDF: 21/09/2015.

\section{RESUMO}

Toxidade do pólen de Ferdinandusa paraensis Ducke (Rubiaceae) para abelhas Apis mellifera L. (Hymenoptera, Apidae) em Roraima, Brasil, região Amazônica. Casos de mortalidade de abelhas pela ingestão de pólen e/ou néctar tóxicos são registrados em vários países. No Brasil, os casos mais severos de mortalidade ocorrem no sudeste, com relatos de perdas anuais de centenas de colônias. Em Roraima existem relatos de mortalidade de abelhas no município de Cantá. A mortalidade é sazonal e ocorre em locais de vegetação preservada sendo que a ingestão por pólen de Ferdinandusa paraensis Ducke pode ser a causa provável. O objetivo deste trabalho foi o de confirmar a toxidade do pólen de F. paraensis para abelhas Apis mellifera L. Foram realizados testes sobrevivência com abelhas operárias engaioladas alimentadas com pólen "in natura", extratos de pólen em hexano, éter etílico, metanol e extratos em etanol de folhas de $F$. paraensis. Tanto o pólen "in natura" quanto extratos de pólen e folhas reduziram significativamente a taxa de sobrevivência para as abelhas operárias $\left(\chi^{2} ; \mathrm{p}<0,0001\right)$. Os resultados deste trabalho confirmam, assim, a toxidade do pólen de $F$. paraensis para abelhas $A$. mellifera $\mathrm{L}$.

PALAVRAS CHAVES: Abelhas melíferas, mortalidade, apicultura.

\section{ABSTRACT}

Pollen Toxicity of Ferdinandusa paraensis Ducke (Rubiaceae) to the bees Apis mellifera L. (Hymenoptera, Apidae) In Roraima, Brazil, Amazon Region. Honeybees mortality by ingestion of pollen and/or nectar toxics are registered in diverse countries. In Brazil, the more severe cases of honeybee mortality occurs in the Southeast, with reports of annual losses of hundreds colonies. In Roraima, in the municipality of Cantá where were reported honeybees mortality cases. The mortality occurs in locals of preserved vegetation probably because the pollen consumption of Ferdinandusa paraensis Ducke. The goal of this work was to confirm the toxicity of $F$. paraensis pollen to honeybees. Survival tests were carried out with trapped worker bees fed with pollen "in natura" and pollen extracts in hexane, methanol, etilic eter and with leaves of Ferdinandusa paraensis. As the pollen "in natura" as its extracts and leaves extracts reduced significantly the survival rate for worker bees $\left(\chi^{2} ; p<0,0001\right)$. These results confirm the pollen toxicity of $F$. paraensis to honeybees (Apis mellifera L.).

KEY WORDS: Honeybees, mortality, bee culture. .

\section{INTRODUÇÃO}

Substâncias tóxicas para as abelhas podem ser encontradas no pólen e/ou no néctar de espécies botânicas. $\mathrm{O}$ efeito desta toxicidade é mais gradual e persiste por um período mais longo, do que nos casos de envenenamento por pesticidas.

Existem muitos casos registrados de envenenamento por plantas tóxicas para as abelhas nos EUA, Canadá e Nova Zelândia. (Root 1990; Atkins 2003). 
No Brasil há vários relatos e estudos sobre plantas que causam intoxicação para as abelhas, dos quais se destacam: Joly (1991), Nogueira Neto (1997), Carvalho \& Message (2004), Cintra et al. (2002, 2005), Pacheco et al. (2009)

Em estudos de sobrevivência é comum a realização de ensaios em laboratório onde abelhas operárias são colocadas em gaiolas e alimentadas com diversos tipos de pólen "in natura" ou seus extratos. Schimidt et al. (1987) testando a sobrevivência de abelhas Apis mellifera L. alimentadas com diversos tipos de pólen em Tucson, Arizona, encontraram uma diferença na longevidade de mais de 40 dias para abelhas alimentadas com pólen de alto valor nutricional. Os autores supõem que a diminuição na longevidade de abelhas, para certos tipos de pólen, esteja relacionada com o baixo teor de proteína, a existência de substâncias deterrentes e a presença de toxidade.

A ocorrência de casos de mortalidade de abelhas $A$. mellifera em Roraima foi registrada por Silva et al. (2002). Abelhas intoxicadas foram encontradas próximas da entrada das colmeias e em casos severos no interior destas.
Esses casos são sazonais e ocorrem em outras localidades, inclusive em áreas preservadas e isentas de atividade agrícola, indicando uma causa natural para essa mortalidade. Silva \& Absy (2005) realizando a análise polínica do conteúdo intestinal de abelhas intoxicadas, apontaram a ingestão de pólen de Ferdinandusa paraensis Ducke (Rubiaceae) como causa provável da mortalidade.

$F$. paraensis é uma árvore de médio a grande porte, ocorrendo em mata primária (Figura 1). Esta espécie é conhecida vulgarmente como "abiu" em Roraima e "bacabinha" no Pará (Amaral et al, 2009). A toxidade para animais de espécies de Rubiaceae vem sendo registrada por vários autores no Brasil: Chiococca alba (L.) por Gazda (2004); Posoqueria acutifolia Mart. por Oliveira et al. (2004) e Sousa et al. (2007); Palicourea marcgravii St.-Hill. por Soto-Blanco et al. (2004) e Silva et al. (2009); Guettarda uruguensis Cham. \& Schltdl. por Duarte (2012); Psychotria colorata ((Willd. ex Schult.) Muell. Arg. por Moura \& Maruo (2014), entre outros. Souza et al. (2013) relacionou 102 estudos etnobotânicos, 20 estudos fitoquímicos
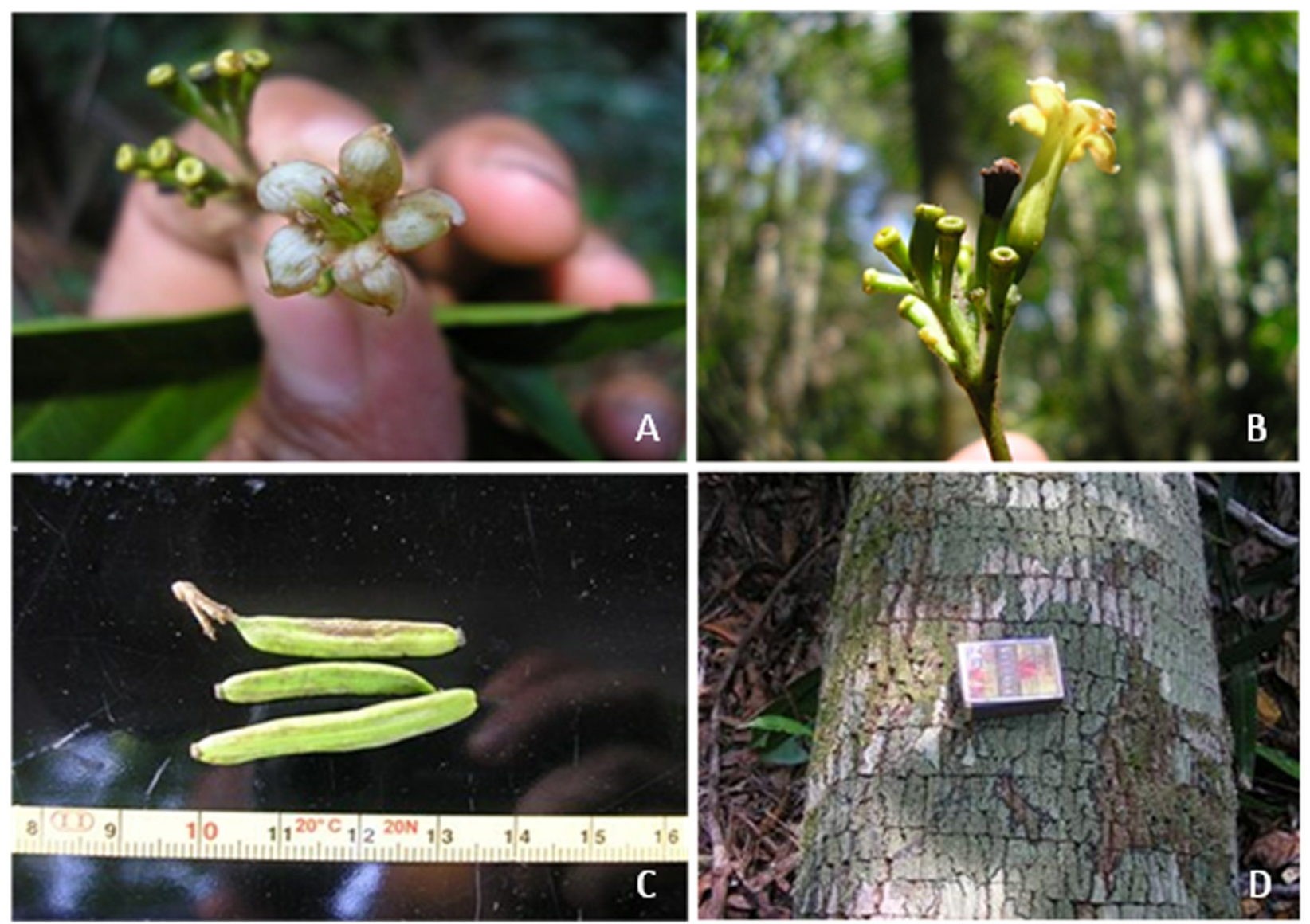

Figura 1. Flores (A e B), frutos (C) e caule (D) de Ferdinandusa paraensis Ducke (Rubiaceae). Fazenda Progresso, Cantá, Roraima. 
e oito estudos farmacológicos com trabalhos relacionados com espécies tóxicas de Rubiaceae no Brasil, coligindo dados de usos, hábitos, indicações, fitoquímica e farmacologia.

Este estudo teve por objetivo confirmar a toxidade de pólen de $F$. paraensis "in natura" e seus extratos para abelhas Apis mellifera L. por meio de testes de toxicidade.

\section{MATERIAL E MÉTODOS}

O pólen utilizado nos testes de toxidade foi obtido por meio de coletores colocados em 15 colmeias de Apis mellifera assentadas na Fazenda Progresso, Município de Cantá, local onde anualmente são registrados casos de intoxicação. De três em três dias durante o período de 30 de outubro de 2002 a 5 de dezembro de 2003, o pólen obtido desses coletores foi recolhido e estocado em freezer para posterior análise polínica. As amostras que continham pólen de $F$. paraensis em percentual superior a $10 \%$ foram selecionadas e separadas para realização dos testes de toxidade.

Os testes de toxidade com o pólen "in natura", seus extratos e extratos de folhas de $F$. paraensis foram realizados em gaiolas de $10 \mathrm{x}$ $10 \times 10 \mathrm{~cm}$ (espaço de confinamento) com tampa de vidro removível, contendo um mínimo de 100 abelhas operárias (Figura 02).

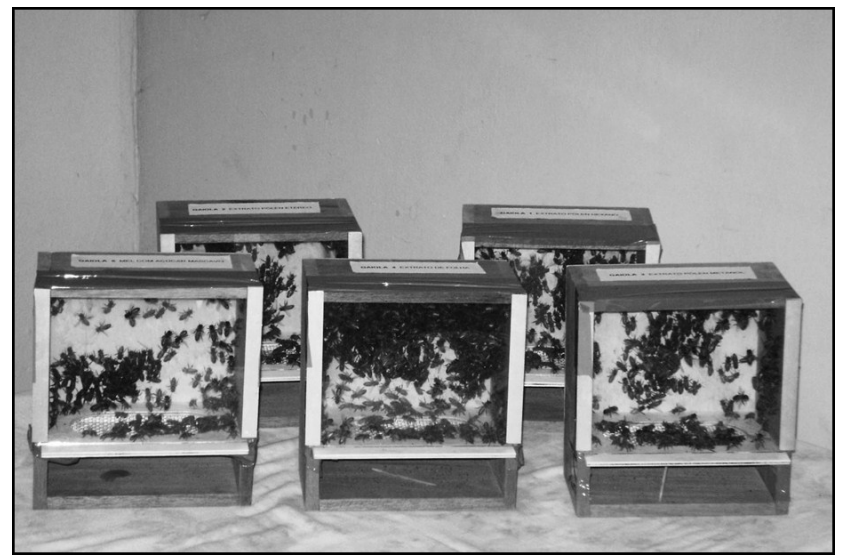

Figura 2. Gaiolas utilizadas nos experimentos de toxidade realizados em Roraima para os testes com pólen "in natura" e seus extratos.

Os testes de toxidade com pólen "in natura" foram realizados no Laboratório de Entomologia do Museu Integrado de Roraima MIRR. O alimento fornecido continha grãos de pólen de $F$. paraensis com percentual variando de $10 \%$ a $30 \%$, os demais tipos polínicos eram principalmente de Mimosa pudica L., Caraipa densifolia Mart., Eschweilera sp., Poaceae spp.,
Attalea maripa (Aubl.) Mart., Mauritia flexuosa L.f. (tratamento). Em outra gaiola (controle) o alimento continha pólen de espécies citadas acima, mas sem a ocorrência de pólen $F$. paraensis, confirmada por meio de análise polínica. Em cada gaiola foram colocadas um mínimo de 100 abelhas operárias coletadas diretamente nos favos de crias. Nas duas gaiolas o alimento foi oferecido como uma pasta de pólen e mel. Os testes de toxidade com pólen "in natura" foram repetidos utilizando-se amostras de datas diferentes, mas com a mesma composição polínica de espécies.

Os testes com extratos foram realizados no Laboratório de Química Fina da Universidade Federal de Roraima - UFRR. Para obtenção dos extratos de pólen foi utilizada a metodologia de Isodorov et al. (2009). A amostra de pólen úmido I5 $(11,47 \mathrm{~g})$ foi extraída sucessivamente com 3 x $100 \mathrm{~mL}$ de hexano, 3x $100 \mathrm{~mL}$ de éter etílico, 3 x $100 \mathrm{~mL}$ de metanol com agitação por ultrassom a temperatura ambiente. A duração de cada extração foi de 30 minutos. As fases orgânicas foram reunidas separadamente de acordo com cada solvente de extração e filtradas utilizando papel de filtro e secas sobre sulfato de sódio. Os solventes foram evaporados em evaporador rotativo e secos em dessecador sob vácuo, obtendo-se um resíduo que foi empregado nos testes de mortalidade de abelhas. Além do pólen também foi testado extratos em etanol de folhas de $F$. paraensis. Os quatro substratos obtidos (pólen/hexano, pólen/éter etílico, pólen/ metanol e folhas/etanol) foram dissolvidos em pasta de açúcar e mel e oferecidos para abelhas engaioladas. Em uma quinta gaiola as abelhas foram alimentadas apenas com pasta de açúcar e mel. Diariamente as abelhas mortas eram retiradas das gaiolas e foram realizadas as contagens dos indivíduos. Ao final dos experimentos se obtinha o total de abelhas engaioladas ( $\mathrm{n}$ amostral). Não foi possível realizar repetições dos testes com extratos devido à pequena quantidade obtida dos mesmos.

A toxidade de $F$. paraensis também foi testada no Centro de Estudos de Insetos Sociais da Universidade Estadual Paulista (UNESP), utilizando-se extrato etéreo de amostras que continham pólen de $F$. paraensis. Foram utilizadas duas gaiolas com abelhas operárias alimentadas com pasta de açúcar e mel. No grupo de tratamento foi adicionado $1 \%$ de extrato e apenas pasta de açúcar e mel no grupo 
controle.

Para a confirmação do efeito tóxico de $F$. paraensis tanto nos testes com pólen "in natura" quanto para os extratos; os resultados foram registrados em tabelas de sobrevivência e para elaboração das curvas de sobrevivência. A análise estatística da toxidade foi realizada através do teste estatístico Log-Rank Test que expressa sua inferência por meio do teste do qui-quadrado $\left(\chi^{2}\right)$.

\section{RESULTADOS E DISCUSSÃO}

Nos testes de toxidade com pólen"in natura", a longevidade das abelhas operárias do grupo de controle foi três vezes maior que do grupo alimentado com pólen de $F$. paraensis (Figura 3A e B).

No gráfico da Figura 3A percebe-se que a
No segundo teste, as diferenças nas taxas de mortalidade foram ainda maiores (Figura 3B). $\mathrm{Na}$ curva de sobrevivência do segundo teste, a mortalidade do grupo que recebeu o pólen de $F$. paraensis continuou acentuada e concentrada nos sete primeiros dias, enquanto que o grupo de controle teve uma longevidade de 27 dias. No grupo de tratamento a sobrevivência foi de cinco dias.

As diferenças de sobrevivência entre tratamento e controle, nos dois testes, foram estatisticamente significativas para o teste do qui-quadrado. No primeiro teste, $F$. paraensis versus controle, o valor de qui-quadrado foi 149,17 com probabilidade $<0,0001$. No segundo teste a essa diferença foi ainda maior com qui-quadrado de 1152,82 e probabilidade $<0,0001$. Também é importante ressaltar que a mortalidade do grupo de tratamento do
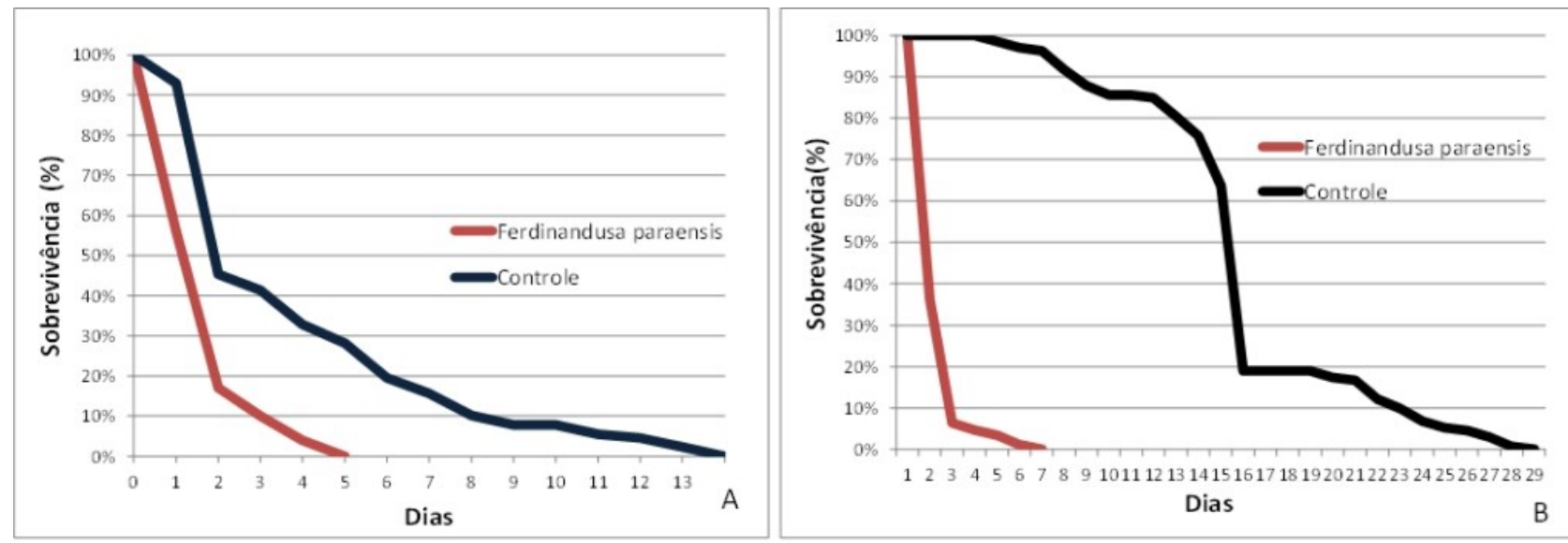

Figura 3. Curvas de sobrevivência para o primeiro (A) e segundo (B) testes de toxidade do pólen "in natura" de Ferdinandusa paraensis.

mortalidade, nos dois primeiros dias, ocorreu tanto no grupo de controle quanto no grupo de tratamento. Este fato, provavelmente se deve a fatores externos como a elevada temperatura ambiente e exposição a luz natural, quando dos registros de mídia (fotos e vídeos) . primeiro teste versus o grupo de tratamento do segundo teste não houve diferenças estatísticas indicando um taxa de mortalidade semelhante (Tabela 01).

Tabela 1. Resultados da análise estatística do qui-quadrado entre o grupo de abelhas com alimento contendo grãos de pólen de Ferdinandusa paraensis "in natura" (tratamento) e o grupo sem a presença de grão de F. paraensis (controle).

\begin{tabular}{ccccc}
\hline Testes & Análise & $\chi^{\mathbf{2}}$ & Probabilidade & Resultado \\
\hline $1^{\circ}$. teste & Ferdinandusa $\times$ Controle & $\mathbf{1 4 9 , 1 7}$ & $<\mathbf{0 , 0 0 0 1}$ & Significativo \\
$2^{\circ}$. teste & Ferdinandusa x Controle & $\mathbf{1 1 5 2 , 8 2}$ & $<\mathbf{0 , 0 0 0 1}$ & Significativo \\
$1^{\circ}$. teste $\times 2^{\circ}$. teste & Ferdinandusa x Ferdinandusa & 3,40 & 0,065 & Não significativo \\
$1^{\circ}$. teste $\times 2^{\circ}$. teste & Controle $\times$ Controle & $\mathbf{5 4 6 , 7 6}$ & $<\mathbf{0 , 0 0 0 1}$ & Significativo \\
\hline
\end{tabular}


Entre o $12^{\circ}$ e $15^{\circ}$ do segundo teste de sobrevivência (Figura 3B) ocorreu grande mortalidade de abelhas operárias no grupo de controle. Curvas de sobrevivência anômalas como as das Figuras 3A e 3B também foram obtidas por Schimidt et al. (1987) testando a sobrevivência de abelhas operárias alimentadas com diversos tipos de pólen.

Nos testes de sobrevivências realizados no Centro de Estudos Sociais da Universidade de São Paulo as diferenças entre as curvas de sobrevivência, para as abelhas alimentadas com extrato etéreo de pólen de $F$. paraensis a 1,0\% (tratamento) e para as abelhas alimentadas apenas com pasta de açúcar somente (controle), não foram acentuadas (Figura 04).

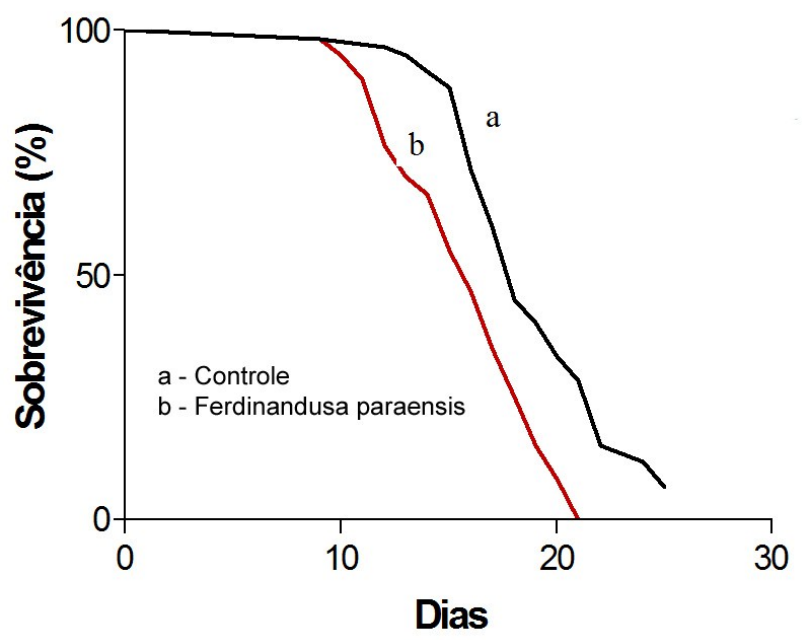

Figura 4. Curva de sobrevivência para teste de toxidade de extrato etéreo de pólen de Ferdinandusa paraensis a $1,0 \%$.

Entretanto essa diferença foi estatisticamente significativa para o teste do qui -quadrado $(p<0,0001)$. Podemos supor que o efeito tóxico não foi tão acentuado, quanto nos testes com pólen "in natura", pois foi utilizado um percentual muito baixo de extrato $(1 \%)$ dissolvido na pasta de açúcar (99\%).

Nas curvas de sobrevivência para os extratos de pólen em hexano, em éter, em metanol e extratos de folhas em etanol de $F$. paraensis, ocorreram diferenças significativas entre os grupos de tratamento e o de controle. O grupo de controle, alimentado apenas com pasta de mel e açúcar, atingiu sete dias de sobrevivência contra apenas três para as abelhas alimentadas com extrato de pólen/hexano e pólen/éter e quatro para os extratos de pólen/metanol e folhas/etanol (Figura 05). Nessas curvas de sobrevivência percebe-se que a mortalidade foi semelhante entre os grupos de abelhas dos tratamentos, tendo o percentual de mortalidade em $100 \%$ no quarto dia do experimento. Houve diferenças percebidas nas curvas de sobrevivência dos tratamentos em comparação com o controle foram confirmadas estatisticamente para o Log-Rank Test na análise do qui-quadrado ( $\mathrm{p}<0,0001$, Tabela 02$)$, confirmando assim o efeito tóxico de $F$. paraensis para as abelhas Apis mellifera.

Tabela 2. Resultado da análise estatística para o teste Log-Rank entre os grupos de abelhas alimentadas com pólen de Ferdinandusa paraensis em extrato em éter etílico, extrato em metanol, extrato em hexano, extrato em etanol de folhas e o grupo de controle.

\begin{tabular}{lcc}
\hline \multicolumn{1}{c}{ TESTE } & $\boldsymbol{\chi}^{\mathbf{2}}$ & Probabilidade \\
\hline Pólen/éter etílico x controle & 392,3 & $<0,0001$ \\
Pólen/metanol x controle & 182,0 & $<0,0001$ \\
Pólen/hexano x controle & 189,3 & $<0,0001$ \\
Folhas/etanol x controle & 235,6 & $<0,0001$ \\
\hline
\end{tabular}

A existência de substâncias tóxicas em $F$. paraensis foi confirmada também por Rodrigues et al. (2014). Os autores confirmaram a toxidade de extratos de partes de $F$. paraensis para fungos patogênicos de Candida spp.

Considerando que todos os extratos de $F$. paraensis realizados neste estudo apresentaram toxidade e que outros estudos também confirmam a toxidade desta planta (Rodrigues et al. 2014) é de se supor que substâncias tóxicas também no pólen sejam encontradas. Para estudos futuros sugere-se a realização de testes de toxidade com o néctar de $F$. paraensis. A determinação do grupo químico e posteriormente da(s) substância(s) envolvidas será objeto de estudo futuro dos autores.

Os resultados obtidos nesse trabalho demonstraram que o pólen de $F$. paraensis "in natura", seus extratos e extratos de folhas foram tóxicos para abelhas operárias de $A$. mellifera. 


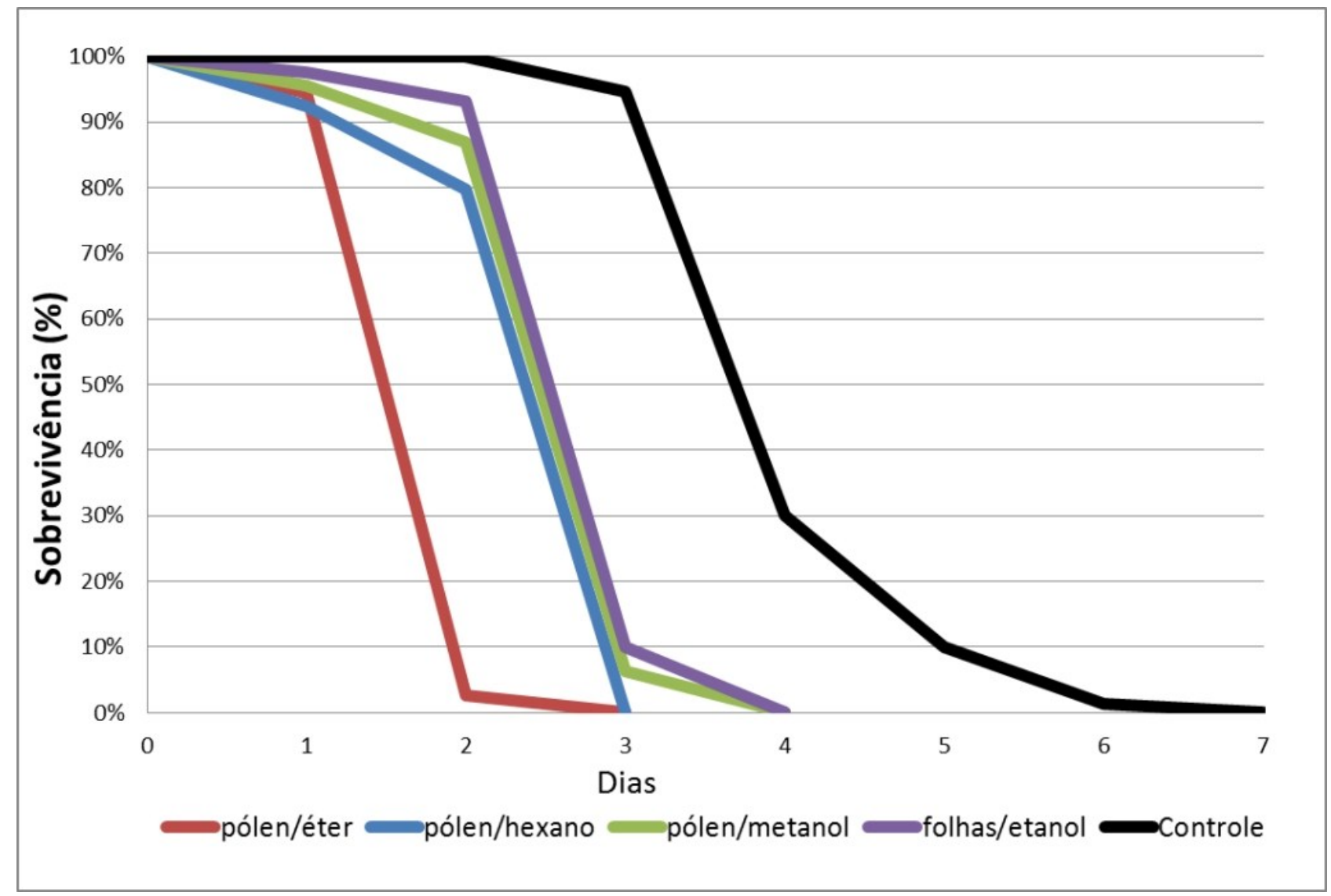

Figura 5. Curva de sobrevivência para testes com abelhas operárias alimentadas com extratos de pólen em hexano, éter etílico, metanol, extrato de folhas em etanol e grupo controle.

\section{REFERÊNCIAS BIBLIOGRÁFICAS}

Amaral, D.D., Vieira, I.C.G., Almeida, S.S. de, Salomão, R. de P., Silva, A.S.L. da \& Jardim, M.A.G. 2009. Checklist da flora arbórea de remanescentes florestais da região metropolitana de Belém e valor histórico dos fragmentos, Pará, Brasil. Boletim do Museu Paraense Emílio Goeldi, série Ciências Naturais 4 (3): 231-289.

Anunciação, E.A. da; Calió, M.F. Ferdinandusa. In: Lista de Espécies da Flora do Brasil. Jardim Botânico do Rio de Janeiro. Disponível em: <http:// floradobrasil.jbrj.gov.br/jabot/floradobrasil/

FB38836>. Acesso em: 07 Ago. 2015>

Atkins, E.L. 2003. Injury to honey bees by poisoning. In: The hive and the honey bee. Dadant \& Sons, Hamilton, Illinois. p.1153-1208.

Brasil, Departamento Nacional de Produção Mineral. 1975. Projeto RADAMBRASIL. v. 8. MME/ Departamento Nacional de Produção Mineral. Rio de Janeiro, 428p.

Carvalho, P.C. \& Message, D. 2004. A scientific note on the toxic pollen of Stryphondenddron polyphyllum (Mimosaceae) which causes sacbrood-like symptoms. Apidologie 35: 89-90.
Cintra, P.; Malaspina, O. \& Bueno, O.C. 2005. Plantas tóxicas para abelhas. Arquivos do Instituto Biológico 72(4):547-551.

Cintra, P.; Malaspina, O.; Petacci, F.; Fernandes, J.B., Bueno, O.C.; Vieira, P.C. \& Silva, M.F.G.F. 2002. Toxity of Dimorphandra mollis to workers of Apis mellifera. Journal of the Brazilian Chemical Society 13(1): 115-118.

Duarte, A.F. S. 2012. Estudo fitoquímico, toxicidade e atividades biológicas: (antioxidante, antimicrobiana e alelopática) de cascas do caule de Guettarda uruguensis Cham \& Schltdl. Rubiaceae. Dissertação de Mestrado. Curitiba: Universidade Federal do Paraná. $117 \mathrm{p}$.

Gazga, V.E. 2004. Abordagem Química e Estudo da Atividade Biológica das Raízes de Chiococca alba (L.) Hitchc. (Rubiaceae). Dissertação de Mestrado. Rio de Janeiro: UFRJ/Faculdade de Farmácia.

Isidorov, V.A.; Isidorova, A.G.; Sczczepaniak L.; Czyzewska, U. 2009. Gas chromatographic-mass spectrometric investigation of the chemical composition of beebread. Food Chemistry 115: 1056 -1063 . 
Joly. A.B. 1991. Botânica: Introdução a taxonomia vegetal. 10ed. Nacional. São Paulo. 777p.

Moura, L.T.S., Maruo, V. M. 2014. Aspectos farmacológicos e toxicológicos de Psychotria colorata - revisão. Revista Científica De Medicina Veterinária 22(23): 1-16.

Nogueira Neto, P. 1997. Vida e criação das abelhas indigenas sem ferrão. São Paulo: Editora Nogueirapis, $445 \mathrm{p}$.

Oliveira, C.M.C. de; Barbosa, J D, Macedo, R.S.C de; Brito, M de F; Peixoto, P V; Tokarnia, C H. 2004. Estudo comparativo da toxidez de Palicourea juruana (Rubiaceae) para búfalos e bovinos. Pesquisa Veterinária Brasileira 24(1): 27-30.

Pacheco, M.R.; Barth, O; M.; Lorenzon, M. C. 2009. Tipos polínicos encontrados em colônias de abelhas africanizadas sujeitas à doença cria ensacada brasileira. Ciência Rural 39(7): 2141-2145.

Ribeiro, J.E.L.S.; Ropkins, M.J.G.; Vicentini, A.; Sother, C.A.; Costa, M.A.; Brito, J.M.; Souza, M.A.D.; Martins, L.H.P; Lohamann, L.G.; Assunção, P.A.C.L.; Pereira, E.C; Silva, C.F.; Mesquita, M.R.; Procópio, L.C. 1999. Flora da reserva Ducke: Guia de identificação das plantas vasculares de uma floresta de terra-firme na Amazônia Central. INPADFID, Manaus. 816p

Rodrigues, K; Ramos, D.F; Carrion, L.L; Cursino, L.M.C.; Jefreys, M.F.; Pedroza. L.S. Osório, M.I.C.; Oliveira. J.L.; Andrade, J.I.A.; Fernandes, C.C.; Nunez, C.V., Silva, P.E.A. 2014. Antifungal activity of Brazilian amazon plants extracts against some species of Candida spp. International Journal of Phytopharmacology 5(6): 445-453.

Root, A.I. Root, E.R. 2010. The $A B C$ and $X Y Z$ of bee culture. A. I. Root Company. Medina, Ohio. 7p.

Schimidt, J.O.; Thoenes, S.C. \& Levin, M.D. 1987. Survival of honey bees, Apis mellifera (Hymenoptera: Apidae) fed various pollen sources. Annals of the Entomogical Soiety of America 80: 175 $-183$.

Silva S.J.R. \& Absy, M.L. 2005. Pólen de Ferdinandusa paraensis Ducke (Rubiaceae) como causa provável da mortalidade de abelhas em Roraima. In: $56^{\circ}$ Congresso Nacional de Botânica, Curitiba (10 a 14 de outubro de 2005).

Silva S.J.R; Absy, M.L.; Casadio, G.M.L. \& Mafra, A.M.S. 2002. Descoberta de pólen tóxico para abelhas (Apis mellifera L.) no estado de Roraima, Brasil. In: ANAIS DO $V$ ENCONTRO SOBRE $A B E L H A S$, 4 a 7 de setembro, Ribeirão Preto - SP. pp. 295.

Silva W.C.; Pinheiro, C.C. de S; Rodrigues, J. M. G. Souza; H.E.M. de S. e Ribeiro, J. D’Arc R. 2009. Avaliação do efeito tóxico de extratos de Palicourea marcgravii St. Hil. (Rubiaceae) sobre Aetalion sp.
(Hemiptera: Aetalionidae) em laboratório. Revista Brasileira de Biociências 7(2): 129-133.

Soto-Blanco, B.; Haraguchi, M.; Silva, J. A.; Górnia, S. L. 2004. Intoxicação natural de caprinos e ovinos por Palicourea marcgravii St. Hil. (Rubiaceae). Revista Caatinga 17(1): 52-56.

Sousa, O.V.; Del-Vechio-Vieira, G.; Almeida, B.H.; Miranda, M.A.; Filgueiras, R.C.; Campos, A.C.; Silvério, M.S. 2007. Efeitos farmacológicos e toxicológicos do extrato de Posoqueria acutifolia Mart. (Rubiaceae) em roedores. Revista de Ciências Farmacêuticas. Básica e Aplicada 28(1): 51-56.

Souza R.K.D., Mendonça A.C.A.M., Silva M.A.P. 2013. Aspectos etnobotánicos, fitoquímicos y farmacológicos de espécies de Rubiaceae en Brasil. Revista Cubana de Plantas Medicinales 18(1):140156. 